Draft Version September 20, 2018

Preprint typeset using $\mathrm{IAT}_{\mathrm{E}} \mathrm{X}$ style emulateapj v. 5/2/11

\title{
HUBBLE SPACE TELESCOPE OBSERVATIONS OF ACCRETION-INDUCED STAR FORMATION IN THE TADPOLE GALAXY KISO 5639
}

\author{
Debra Meloy Elmegreen ${ }^{1}$, Bruce G. Elmegreen ${ }^{2}$, Jorge Sánchez Almeida ${ }^{3}$, Casiana Muñoz-Tuñón ${ }^{3}$, Jairo \\ Mendez-Abreu ${ }^{4}$, John S. Gallagher ${ }^{5}$, Marc Rafelski $^{6}$, Mercedes Filho ${ }^{3}$, Daniel Ceverino ${ }^{7}$ \\ Draft version September 20, 2018
}

\begin{abstract}
The tadpole galaxy Kiso 5639 has a slowly rotating disk with a drop in metallicity at its star-forming head, suggesting that star formation was triggered by the accretion of metal-poor gas. We present multi-wavelength HST WFC3 images of $U V$ through $I$ band plus $\mathrm{H} \alpha$ to search for peripheral emission and determine the properties of various regions. The head has a mass in young stars of $\sim 10^{6} M_{\odot}$ and an ionization rate of $6.4 \times 10^{51} \mathrm{~s}^{-1}$, equivalent to $\sim 2100$ O9-type stars. There are four older star-forming regions in the tail, and an underlying disk with a photometric age of $\sim 1$ Gyr. The mass distribution function of 61 star clusters is a power law with a slope of $-1.73 \pm 0.51$. Fourteen young clusters in the head are more massive than $10^{4} M_{\odot}$, suggesting a clustering fraction of $30 \%-45 \%$. Wispy filaments of $\mathrm{H} \alpha$ emission and young stars extend away from the galaxy. Shells and holes in the head Hil region could be from winds and supernovae. Gravity from the disk should limit the expansion of the HiI region, although hot gas might escape through the holes. The star formation surface density determined from $\mathrm{H} \alpha$ in the head is compared to that expected from likely pre-existing and accreted gas. Unless the surface density of the accreted gas is a factor of $\sim 3$ or more larger than what was in the galaxy before, the star formation rate has to exceed the usual Kennicutt-Schmidt rate by a factor of $\geq 5$.

Subject headings: galaxies: star formation- galaxies: photometry - galaxies: dwarf - galaxies: star clusters
\end{abstract}

\section{INTRODUCTION}

Tadpole galaxies are characterized by a bright starforming head and an elongated tail with weak star formation. They are sometimes called cometary (Markarian 1969, Loose \& Thuan 1985, Cairós et al. 2001) and are designated sub-class il,c of the Blue Compact Dwarf (BCD) type by Noeske et al. (2000). Other local tadpoles were studied by Kniazev et al. (2001), Gil de Paz, Madore, \& Pevunova (2003), Papaderos et al. (2008), Elmegreen et al. (2012), and others. They are rare in the local universe, where only $0.2 \%$ of the 10,000 galaxies in the Kiso Survey of UV Bright Galaxies (Miyauchi-Isobe, Maehara, \& Nakajima 2010) are tadpoles (Elmegreen et al. 2012), but they are common, $75 \%$ (Morales-Luis et al. 2011), among local extremely metal poor (XMP) galaxies, which have metallicities less than 0.1 solar.

The association between tadpole shapes and BCD or low metallicity galaxies suggests an element of youthfulness. Indeed, tadpoles are relatively common in the

\footnotetext{
${ }^{1}$ Vassar College, Dept. of Physics and Astronomy, Poughkeepsie, NY 12604

${ }^{2}$ IBM Research Division, T.J. Watson Research Center, Yorktown Hts., NY 10598

3 Instituto de Astrofísica de Canarias, C/ via Láctea, s/n, 38205, La Laguna, Tenerife, Spain, and Departamento de Astrofísica, Universidad de La Laguna

${ }^{4}$ School of Physics \& Astronomy, North Haugh, St Andrews, KY169SS, UK

${ }^{5}$ Dept. of Astronomy, Univ. of Wisconsin-Madison, Madison, WI 53706

${ }^{6}$ NASA Postdoctoral Program Fellow, Astrophysics Science Division, Goddard Space Flight Center, Code 665, Greenbelt, MD 20771

${ }^{7}$ Zentrum für Astronomie der Universität Heidelberg, Institut für Theoretische Astrophysik, Albert-Ueberle-Str. 2, 69120 Heidelberg, Germany
}

early Universe, amounting to some $10 \%$ of all galaxies larger than 10 pixels in diameter in the Hubble Space Telescope Ultra Deep Field (Elmegreen et al. 2005), and a similar fraction in other deep fields (van den Bergh et al. 1996, Straughn et al. 2006, Windhorst et al. 2006). XMP galaxies are also gas-rich, giving them a low average HI star formation efficiency (= star formation surface density/HI surface density), but they have relatively few stars, so their specific star formation rates (SFR/stellar mass) are high, comparable to those in high redshift galaxies (Filho et al. 2015).

Single slit spectra of 22 tadpoles or XMP galaxies have been used to determine metallicities along the major axes and, in a few cases, rotation curves. Sánchez Almeida et al. (2013) obtained spectra of 7 tadpoles with the $2.5 \mathrm{~m}$ Isaac Newton Telescope and the $2.5 \mathrm{~m}$ Nordic Optical Telescope on Tenerife. Rotation was observed, with a peak speed of several tens of $\mathrm{km} \mathrm{s}^{-1}$, as expected for these low mass objects. The metallicities were unusual, however. As inferred from the $\mathrm{N} 2$ index (the ratio [N $\mathrm{II}] / \mathrm{H} \alpha$ ), the metallicities were lowest in the star-forming heads in 6 out of the 7 cases. Sánchez Almeida et al. (2014a) determined metallicities in 7 more dwarf galaxies with the $4.2 \mathrm{~m}$ William Hershel Telescope using the "direct method" (i.e., [SII] lines $\lambda 6717 / \lambda 6731$ for electron density, [OIII] lines $(\lambda 4959+\lambda 5007) / \lambda 4363$ for electron temperature of $[\mathrm{OIII}]$, and $[\mathrm{OII}]$ lines $\lambda 3727 /(\lambda 7319+$ $\lambda 7330)$ for electron temperature of [OII], with a sum of the oxygen states up to [OIII] to give the oxygen abundance relative to hydrogen). Of these 7 galaxies, 2 out of 5 with measurable metallicities had low values in the head; the other 2 were ambiguous. Spectra of another 10 XMPs, including tadpoles, were obtained with the $10.4 \mathrm{~m}$ Gran Telescopio Canarias using the HII-CHI-mistry al- 
Table 1

HST WFC3 Observations

\begin{tabular}{|c|c|c|c|c|}
\hline $\begin{array}{c}\mathrm{RA} \\
(\mathrm{J} 2000)\end{array}$ & $\begin{array}{c}\text { Dec } \\
\text { J2000 }\end{array}$ & filter & $\begin{array}{c}\text { Exposure time } \\
(\mathrm{sec})\end{array}$ & Date \\
\hline 114109.410 & +322527.21 & F225W & 2508.0 & $02-16-2015$ \\
\hline 114109.410 & $\begin{array}{r}+322527.21 \\
\end{array}$ & F336W & 2508.0 & $02-16-2015$ \\
\hline 114108.702 & +322540.21 & F438W & 1248.0 & $07-02-2015$ \\
\hline 114108.702 & +322540.21 & $\mathrm{~F} 547 \mathrm{M}$ & 1248.0 & $07-02-2015$ \\
\hline 114108.702 & +322540.21 & F606W & 1310.0 & $07-02-2015$ \\
\hline 114108.702 & +322540.21 & F814W & 1310.0 & $07-02-2015$ \\
\hline 114108.640 & +322541.13 & F657N & 2624.0 & $07-02-2015$ \\
\hline
\end{tabular}

gorithm (Pérez-Montero 2014 i.e., minimizing the $\chi^{2}$ fit to many lines). In 9 out of 10 cases, the metallicities were lower in the heads by factors of 3 to 10 (Sánchez Almeida et al. 2015).

We interpret these metallicity drops as the result of recent accretion of metal-poor gas onto part of an otherwise quiescent dwarf galaxy. Idealized simulations (Verbeke et al. 2014) as well as cosmological simulations of galaxy formation (Ceverino et al. 2016) show this effect. A recent review of accretion-fed star formation is discussed in Sánchez Almeida et al. (2014b). Support for this interpretation comes from the locations of most of the XMPs in low-density environments, such as voids and sheets in the distribution of galaxies (Filho et al. 2015). They are surrounded by low-metallicity, oftenasymmetric Hi (Filho et al. 2013), which could be the accreting gas reservoir. Motion through this reservoir could cause the accretion and promote star formation on the leading edge of the dwarf, as might have happened for 30 Doradus in the Large Magellanic Cloud (Mastropietro et al. 2009). High-mass late-type galaxies could also be bursting with star formation in their outer parts from episodic accretion (Huang et al. 2013).

One of the best examples of a metallicity drop is in the bright star-forming head of the local tadpole galaxy Kiso 5639, which is the focus of the present paper. Here we examine Hubble Space Telescope (HST) Wide Field Camera 3 (WFC3) multiwavelength images of Kiso 5639 to determine the masses and ages of the clusters and large-scale star-forming clumps, and to search for evidence of accretion on the periphery. Our data are presented in Section 2, analyses are in Section 3, results are in Section 4, and conclusions are in Section 5 .

\section{OBSERVATIONS AND DATA REDUCTION}

Kiso 5639 (LEDA-36252, KUG 1138+327) is a small emission line galaxy approximately $2.7 \mathrm{kpc}$ in diameter at a galactocentric distance of $24.5 \mathrm{Mpc}\left(\mathrm{NED}^{8}\right)$. It is slowly rotating with a velocity of $35-40 \mathrm{~km} \mathrm{~s}^{-1}$, giving it a dynamical mass of $1.5 \times 10^{8} M_{\odot}$ (Sánchez Almeida et al. 2013 ). Its photometric mass is $5 \times 10^{7} M_{\odot}$ based on Sloan Digital Sky Survey images (Elmegreen et al. 2012), and the HI mass is around $3 \times 10^{8} M_{\odot}$ (Salzer et al. 2002, see Sect. 4.4. HST WFC3/UVIS images of Kiso 5639 were obtained in February and July 2015 (Proposal 13723) in filters F225W, F336W, F438W, F547M, F606W, F814W, and $\mathrm{F} 657 \mathrm{~N}(\mathrm{H} \alpha+\mathrm{N} \mathrm{II})$, where the parentheses indicate wide $(\mathrm{W})$, medium $(\mathrm{M})$, and narrow $(\mathrm{N})$ filters with cen-

8 NASA/IPAC Extragalactic Database, http://ned.ipac. caltech.edu tral wavelengths in nanometers; exposure times and coordinates for the observations are listed in Table 1. At the assumed distance, the pixel size of 0.0396 arcsec corresponds to $4.7 \mathrm{pc}$. We placed the galaxy near the edge of the detector to put it close to the read-out to minimize charge transfer efficiency (CTE) degradation effects, and adjusted its position with the F225W and F336W filters to avoid a bright star.

The WFC3/UVIS instrument has degraded over time due to radiation damage, reducing the CTE, which results in smeared images along the read-out direction when not corrected (MacKenty \& Smith 2012). The science images are therefore CTE corrected using code provided by STSc $\sqrt{9}$. However, the dark calibrations also suffer from this degradation, and therefore we required special calibration files to mask all the hot pixels, and remove a background gradient and splotchy pattern in the science images. To do so, we created custom super-darks following the method described in Rafelski et al. (2015), which use all CTE corrected post-Hash darks from an anneal cycle to determine the average dark level per good pixel, and mark all hot pixels on shorter timescales with a uniform threshold. This resulted in cleaner and more accurate images, especially for near ultra-violet and narrow-band images. The CTE-corrected and newly calibrated science exposures were then combined using AstroDrizzle and an 0.8 pixel fraction at the original pixel scale.

Figure 1 shows the images for the individual filters and for the continuum-subtracted $\mathrm{H} \alpha$ image. The latter was made by scaling the average counts of the F547M image to the F657N image using the ratio of the PHOTFLAM value 10 for the two filters. PHOTFLAM is the inverse sensitivity, given in units of $\mathrm{erg} \mathrm{cm}^{-2} \AA^{-1}$ per electron count. In practice, this means that the F547M image was divided by 4.78 and then subtracted from the F657N image to make the continuum-subtracted $\mathrm{H} \alpha$ image. We used the F547M filter for this rather than the F606W filter because the $\mathrm{F} 606 \mathrm{~W}$ filter contains the $\mathrm{H} \alpha$ line and an equally strong [OIII] line at $5008 \stackrel{\circ}{A}$ according to the spectrum of this galaxy in the SDSS. The F547M filter lies between these lines.

Color composite figures were made in the Image Reduction and Analysis Facility (IRAF) using RGB in DS9. Figure 2 shows F438W as blue, F547M as green, and $\mathrm{F} 657 \mathrm{~N}$ as red (which is $\mathrm{H} \alpha$ including the continuum). The line indicates $500 \mathrm{pc}$, corresponding to $4.2^{\prime \prime}$. The periphery of the galaxy is outlined by red wispy protru-

\footnotetext{
9 http://www.stsci.edu/hst/wfc3/tools/cte_tools

10 http://www.stsci.edu/hst/wfc3/phot_zp_lbn
} 


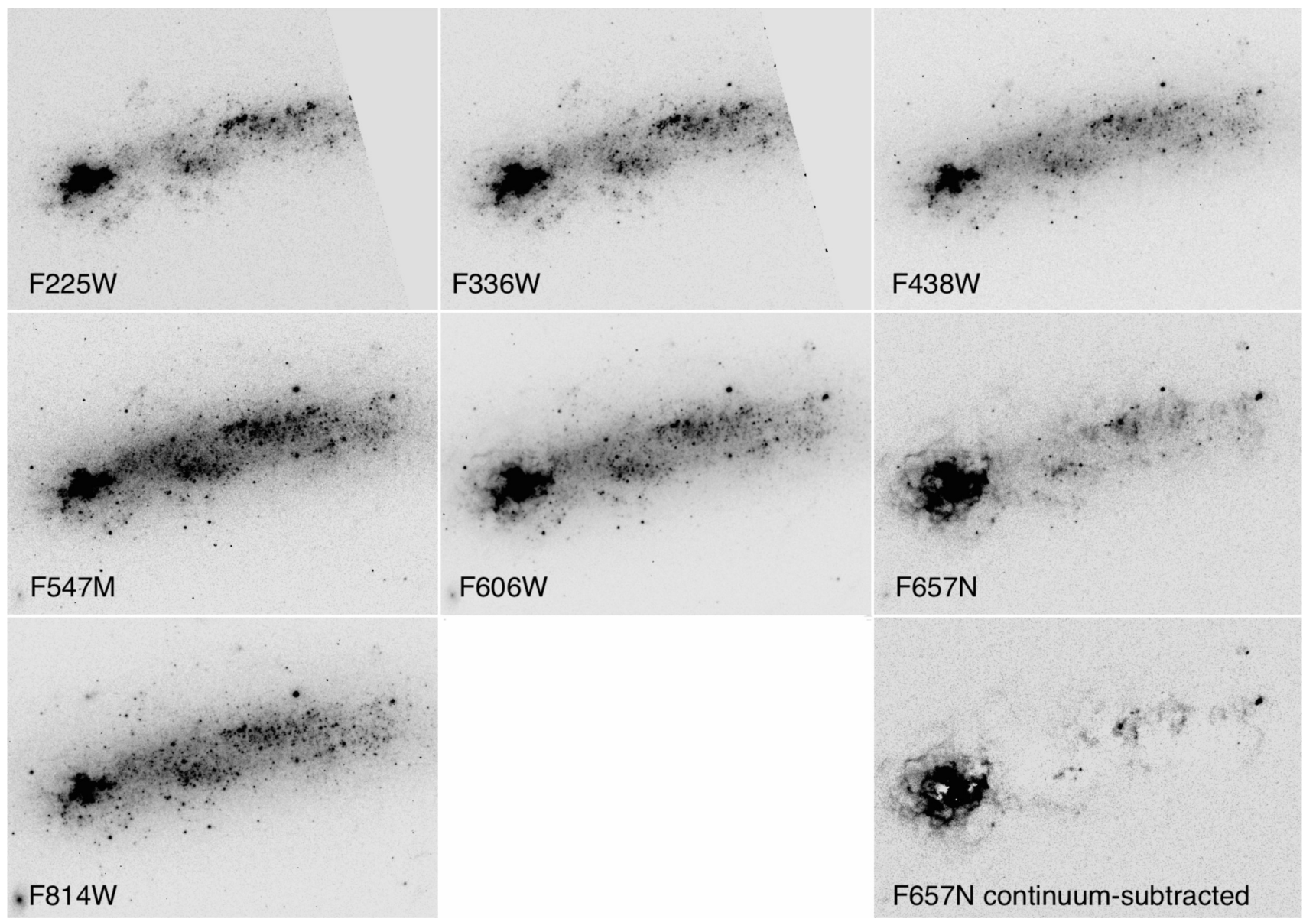

Figure 1. Kiso 5639 images from HST WFC3 observations, with filters indicated. The lower right-hand figure is the continuum-subtracted H $\alpha$ image. $\mathrm{N}$ is up, E to the left.

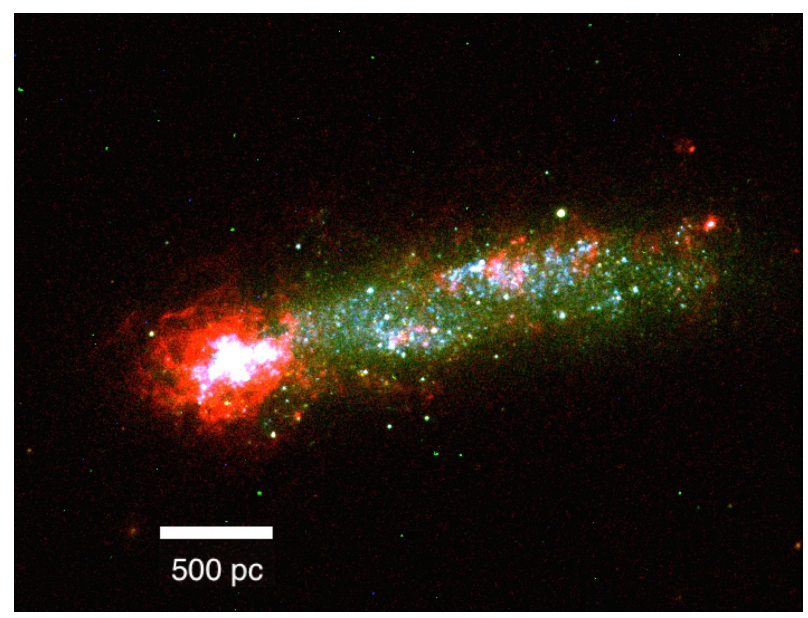

Figure 2. Kiso 5639 color composite image from F657N (in red), F547M (in green), and F438W (in blue) filters. The line indicates $500 \mathrm{pc}$, corresponding to $4.2^{\prime \prime}$. $\mathrm{N}$ is up, E to the left.

sions from $\mathrm{H} \alpha$, discussed further in Section 4 These wisps are shown better in Figure 3 , which is F657N on a linear scale, Gaussian smoothed by a factor of 5 in DS9 to enhance the faint structures.

Figure 4 shows a large field of view with $\mathrm{F} 438 \mathrm{~W}$ as blue, F547M as green, and F814W as red. Small background galaxies can be seen, including a larger galaxy with an unknown redshift to the NE of Kiso 5639. The

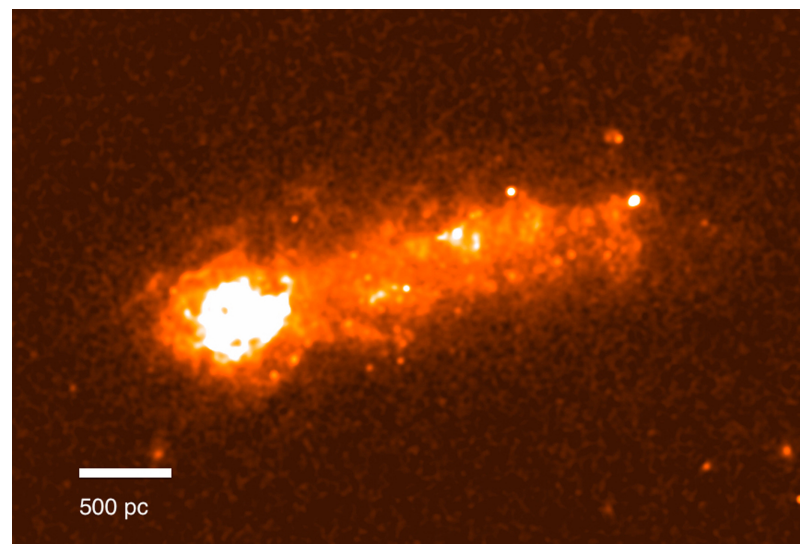

Figure 3. False color image of Kiso 5639 in $\mathrm{H} \alpha$ (F657N) (not continuum-subtracted) on a linear scale, Gaussian smoothed by a factor of 5 in DS9 to enhance the faint peripheral gas emission. The line indicates $500 \mathrm{pc}$, corresponding to $4.2^{\prime \prime}$.

image is shown on a logarithmic scale to enhance the extended envelope around the main body of Kiso 5639. Note the extended oval of yellow color, which is interpreted in Section 4.2 as an underlying disk. There is also a small network of intersecting filaments in the north, one-third of the way along the galaxy from the head to the tail.

The head region is shown enlarged in Figure 5, with F438W in blue, F547M in green, and F814W in red. 


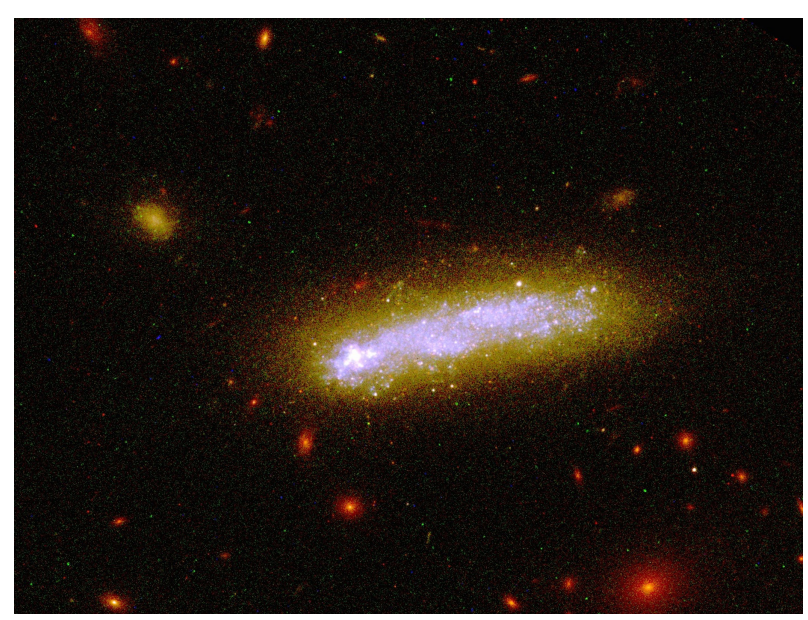

Figure 4. Large field around Kiso 5639 in a color composite image from $\mathrm{F} 438 \mathrm{~W}$ (in blue), F547M (in green), and F814W (in red) filters, shown on a logarithmic scale to enhance the outer boundaries. Note the small cluster of galaxies surrounding it, and a small galaxy to the northeast. $\mathrm{N}$ is up, $\mathrm{E}$ to the left.

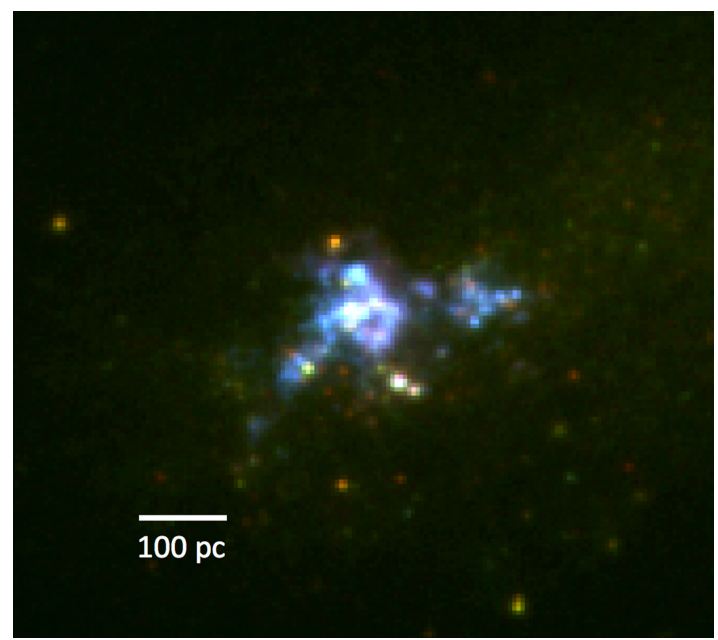

Figure 5. The head region of Kiso 5639 is shown in a color composite image with F336W (blue), F547M (green), and F814W (red). The white line corresponds to $100 \mathrm{pc}=21.2 \mathrm{px}=0.84^{\prime \prime}$. The central white cluster is the youngest and most massive in the galaxy.

The line indicates $100 \mathrm{pc}$, or $\sim 0.8^{\prime \prime}$. There are many bright clusters and discrete regions of star formation that are described in the following sections. In Figure 6, the $\mathrm{H} \alpha$ continuum-subtracted image is shown in red, with F547M in green and F225W in blue, to highlight the juxtaposition of young clusters with the $\mathrm{H} \alpha$. There are many cavities in the HII region (Sect. 4.3.5.

\section{ANALYSIS}

Photometric measurements were made of star-forming regions for each image using the task imstat in IRAF. Boxes were defined around the 5 most prominent largescale clumps, 100-200 pc in diameter, based on isophotal contours in the F438W image. One clump is the bright part of the head and four are in the tail. These 5 regions are identified as blue ellipses in Figure 7 and labeled $a$ through $e$. The average interclump background was subtracted from each measurement to get the magnitudes of the individual regions. The interclump surface bright-

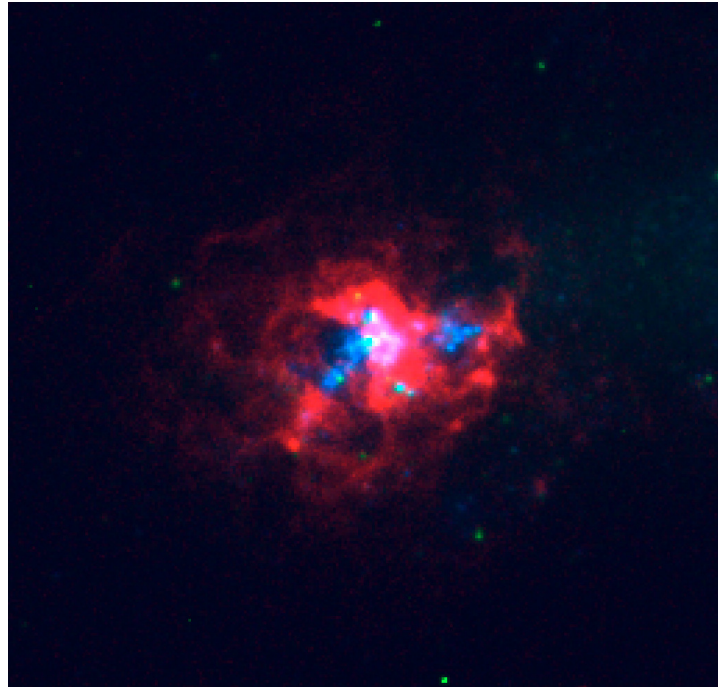

Figure 6. The head region Kiso 5639 is shown in a color composite image with F225W (blue), F547M (green) and continuumsubtracted $\mathrm{H} \alpha$ (red) to highlight the structure of the emission relative to the young central clusters.

nesses in regions $f$ through $i$ were also determined. Photometric zeropoints from the WFC3 website 11 were used to convert counts to $\mathrm{AB}$ mag.

In addition, 61 individual clusters or unresolved complexes $10-20$ pc in size were measured in all of these regions, selected by eye from the F438W images. These clusters are brighter than $25 \mathrm{AB}$ mag in the F438W image. The average interclump background was subtracted from each of these. Ten filamentary regions extending away from the bright part of the galaxy were measured too, as outlined in Figure 8, with no background subtraction.

Masses and ages were determined by fitting photometric magnitudes and colors to population synthesis models from Bruzual \& Charlot (2003) with wavelengthdependent extinction laws from Calzetti et al. (2000) and Leitherer et al. (2002). We followed the procedures in Elmegreen et al. (2012) and previous papers, using the

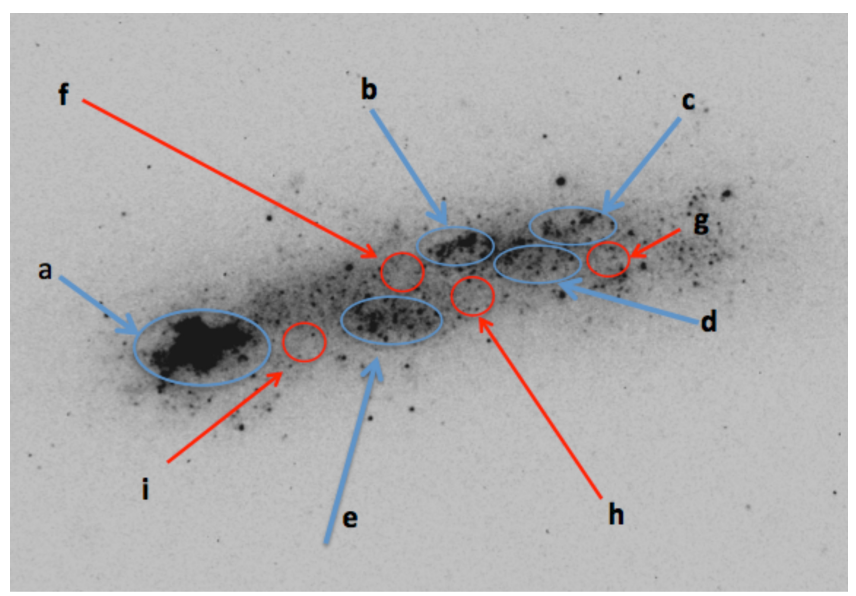

Figure 7. The 5 main regions in Kiso 5639, plus 4 interclump regions, are indicated on the $\mathrm{F} 438 \mathrm{~W}$ image. The head is more massive and younger than the other regions; the interclump regions are the oldest. Their properties are listed in Table 2

11 http://www.stsci.edu/hst/wfc3/phot_zp_lbn 


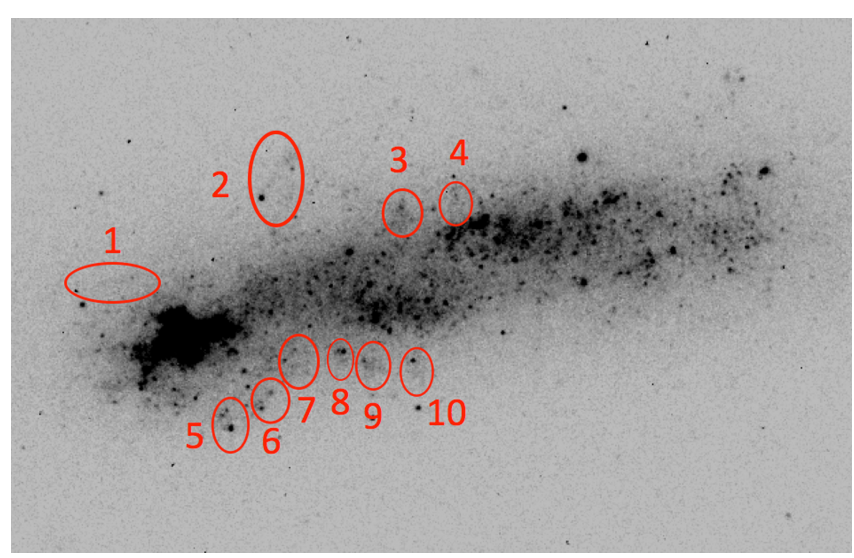

Figure 8. Locations of 10 peripheral filaments or emission regions in Kiso 5639 on the F438W image, as seen more directly in Figures 23 and 4 The average masses and ages are in Table 2.

integrated population spectra as a function of time as tabulated in Bruzual \& Charlot (2003), and the UVIS filters on HST. Throughputs for the UVIS filters were from the WFC3 website 12 . The Chabrier initial mass function (IMF) and a metallicity of 0.2 solar were assumed. For the individual clusters, color models were generated, with the age of the cluster corresponding to the beginning of star formation, and an exponential decay going forward. Models for the large star-forming regions and the interclump regions were made assuming a constant SFR back to the fitted age.

Many models were run for each region, covering all combinations of visual extinction from 0 to $7.5 \mathrm{mag}$ in steps of $0.25 \mathrm{mag}$, logarithmic age (in years) in 50 equal steps from 5.1 to 10, and the decay times of $1,3,10$, 30 and 100 times $10^{7}$ years. Each combination produced theoretical colors that were compared to the observed colors using the quadratic sum of differences, $\delta^{2}$. This $\delta^{2}$ is different from $\chi^{2}$ by not having the rms noise in the denominator of each difference. The rms noise from observations is much less than the rms variation from model to model. The region masses were determined from the models for each case also, scaling the theoretical magnitude in F438W to the observed magnitude and using the masses tabulated for each population and age by Bruzual \& Charlot (2003). We then picked as reasonable solutions those with the lowest $\delta^{2}$, and among those took the averages of the masses, ages, extinctions and decay times using a weighting function of $\exp \left(-0.5 \delta^{2}\right)$.

The metallicity measurements of Kiso 5639 by Sánchez Almeida et al. (2013) are 0.1 solar for the head and 0.2 solar for the tail, so the tabulated Bruzual models with a metallicity of 0.2 solar $(\mathrm{Z}=0.004)$ are reasonable. To determine how important metallicity was, solutions for the individual clusters were also found for solar and 0.4 solar metallicities. For the 21 head clusters, the log-masses were on average $4.2 \%$ and $1.5 \%$ smaller than our measurements for solar and 0.4 solar metallicity, their logages were $3.9 \%$ and $3.4 \%$ larger, their extinctions were $16.6 \%$ and $9.2 \%$ smaller, and their SFR decay log-times were less than $1 \%$ larger, respectively. These changes are small enough to ignore metallicity effects on the photo-

12

http://www.stsci.edu/hst/wfc3/ins_performance/ throughputs/Throughput_Tables metric analysis.

Star formation rates (SFRs) were determined from the continuum-subtracted $\mathrm{H} \alpha$ image for the large-scale regions. Taking into account the distance and the filter width $(121 \AA)$, the luminosity is given by $L(H \alpha)=$ $2.19 \times 10^{46} \times 10^{-0.4 m_{\alpha}}$ erg $\mathrm{s}^{-1}$ for apparent $\mathrm{AB} \mathrm{H} \alpha$ magnitude, $m_{\alpha}$. 13 The SFRs were determined from $S F R=7.9 \times 10^{-42} L(H \alpha) M_{\odot} \mathrm{yr}^{-1}$ using the calibration from Kennicutt (1998).

\section{RESULTS}

\subsection{Masses, Ages, and Star formation rates}

Table 2 lists the mass and age solutions from the photometry for the main large-scale star-forming clumps in the head and tail (regions $a-e$ in Figure 7), for the averages of all the individual clusters in the head and tail regions, for the interclump regions $(f-i)$, and for the averages of the filamentary protrusions on the periphery of the galaxy in the head (ellipse 1 in Figure 8) and in the tail (ellipses $2-9$ ). The SFRs are the ratios of the photometric masses to the ages, the surface densities are the ratios of the masses to the areas used for the photometry, and the $\mathrm{H} \alpha$ luminosities and $\mathrm{H} \alpha$ SFRs are from the continuum-subtracted $\mathrm{H} \alpha$ filter, evaluated only for the large regions.

Uncertainties for the solutions were determined from the three sources of uncertainty added in quadrature. The first is the dispersion in the best fit log-values for each cluster in a region. The second is the variation in the model results (in logarithms) among all of the individual solutions for each cluster that were combined with the $\exp \left(-0.5 \delta^{2}\right)$ weighting factor to give the best fit (see above). The third is the measurement error from the standard deviation of the image counts relative to the average counts in each region, summed in quadrature for each filter.

Extinction results are not given in the table but they average about $0.6-0.8 \mathrm{mag}$ visual for all of the clusters in the head and tail. The average decay rate in the best fit cluster solutions is $\sim 10^{8}$ years, which is typically longer than the cluster age, meaning that the preferred SFRs are fairly constant.

Figure 9 shows histograms for mass, age, and SFR for the 21 clusters in the head (top panels) and the 40 clusters in the 4 main clumps in the tail (regions $b-e$ ). The individual clusters in the head have average masses of $1.6 \times 10^{4} M_{\odot}$, which is 3-6 times larger than those in the tail. The ages of the head clusters average $\sim 8 \times 10^{5} \mathrm{yr}$, or about 7-12 times younger than in the tail.

A histogram of mass for all 61 measured clusters is shown in Figure 10 with a power law fit at $\log M \geq 3.5$. The detection limit is $\sim 3 \times 10^{3} M_{\odot}$. The slope of the mass function is $-0.73 \pm 0.51$ on this log-log plot, which corresponds to a slope of $-1.73 \pm 0.51$ in linear intervals of mass, i.e., $d N / d M \propto M^{-1.73 \pm 0.51}$. The error bars are the $90 \%$ confidence interval for a student t test with 4 degrees of freedom. The correlation coefficient is $r=0.99$. This slope is typical for cluster mass functions (Larsen 2009).

Star-forming regions in galaxies generally follow a hierarchical size distribution (Elmegreen 2010). To check

13 The coefficient is $4 \pi D^{2} \Delta \lambda c / \lambda^{2} 10^{-0.4 \times 48.6}$ for distance D, filter width $\Delta \lambda$, speed of light $c$ and wavelength $\lambda$. 
Table 2

Masses, Ages, and Star Formation Rates

\begin{tabular}{|c|c|c|c|c|c|c|}
\hline Region & $\begin{array}{c}\log \text { Mass } \\
\left(\mathrm{M}_{\odot}\right)\end{array}$ & $\begin{array}{c}\log \text { Age } \\
(y r)\end{array}$ & $\begin{array}{c}\log \mathrm{SFR} \\
\left(\mathrm{M}_{\odot} \mathrm{yr}^{-1}\right) \\
\end{array}$ & $\begin{array}{l}\text { Surface density } \\
\quad\left(\mathrm{M}_{\odot} \mathrm{pc}^{-2}\right)\end{array}$ & $\begin{array}{c}\mathrm{L}(\mathrm{H} \alpha) \\
\times 10^{37} \mathrm{erg} \mathrm{s}^{-1}\end{array}$ & $\begin{array}{c}\log \operatorname{SFR}(\mathrm{H} \alpha) \\
\left(\mathrm{M}_{\odot} \mathrm{yr}^{-1}\right)\end{array}$ \\
\hline \multicolumn{7}{|l|}{ Main clumps ${ }^{\mathrm{a}, \mathrm{b}}$ : } \\
\hline b (tail) & $5.2 \pm 0.14$ & $6.6 \pm 0.23$ & $-1.4 \pm 0.27$ & 4.3 & $13 \pm 0.1$ & -3.0 \\
\hline c (tail) & $5.0 \pm 0.10$ & $5.8 \pm 0.64$ & $-0.7 \pm 0.64$ & 2.9 & $14 \pm 0.3$ & -3.0 \\
\hline d (tail) & $5.2 \pm 0.10$ & $6.7 \pm 0.05$ & $-1.5 \pm 0.02$ & 3.1 & $8.6 \pm 0.2$ & -3.2 \\
\hline e (tail) & $5.6 \pm 0.11$ & $7.5 \pm 0.63$ & $-1.9 \pm 0.64$ & 3.0 & $18 \pm 0.3$ & -2.8 \\
\hline \multicolumn{7}{|l|}{ Clusters $^{\mathrm{C}}$ : } \\
\hline a (head, 21) & $4.2 \pm 0.28$ & $5.9 \pm 0.88$ & $-1.7 \pm 0.92$ & $66 \pm 25$ & & \\
\hline b (tail, 10) & $3.7 \pm 0.46$ & $7.0 \pm 0.93$ & $-3.3 \pm 1.04$ & $23 \pm 18$ & & \\
\hline c (tail, 9) & $3.5 \pm 0.32$ & $6.8 \pm 0.74$ & $-3.4 \pm 0.80$ & $15 \pm 5.7$ & & \\
\hline d (tail, 9) & $3.5 \pm 0.36$ & $6.7 \pm 0.78$ & $-3.3 \pm 0.86$ & $16 \pm 7.4$ & & \\
\hline e (tail, 12) & $3.5 \pm 0.48$ & $6.7 \pm 0.95$ & $-3.2 \pm 1.07$ & $43 \pm 77$ & & \\
\hline \multicolumn{7}{|l|}{ Interclumps $^{\mathrm{d}}$ : } \\
\hline $\mathrm{f}$ & $5.2 \pm 0.07$ & $8.9 \pm 0.51$ & $-3.7 \pm 0.52$ & 26.3 & & \\
\hline $\mathrm{g}$ & $4.9 \pm 0.03$ & $9.0 \pm 0.39$ & $-4.0 \pm 0.39$ & 18.2 & & \\
\hline $\mathrm{h}$ & $5.3 \pm 0.09$ & $9.1 \pm 0.55$ & $-3.8 \pm 0.56$ & 29.8 & & \\
\hline & $5.6 \pm 0.03$ & $9.1 \pm 0.36$ & $-3.5 \pm 0.36$ & 28.3 & & \\
\hline \multicolumn{7}{|l|}{ Periphery ${ }^{\mathrm{e}}$ : } \\
\hline 1 (head) & $4.6 \pm 0.12$ & $5.8 \pm 0.65$ & $-1.2 \pm 0.66$ & 8.7 & & \\
\hline $2-10$ (tail) & $4.5 \pm 0.42$ & $7.6 \pm 0.89$ & $-3.1 \pm 0.98$ & $5.8 \pm 2.7$ & & \\
\hline
\end{tabular}

a SED fits for the head removed line emission from the F606W flux. A constant star formation rate back to the indicated age was assumed for these large-scale regions.

$\mathrm{b}$ The masses and ages for the clump regions correspond to rectangles with areas of $8085,1512,1566,2160$, and 5252 pixels for a, b, c, d, and e, respectively. The SED fits are an average of the fits with the lowest rms deviations from the observed colors considering exponentially decaying star formation rates starting at the indicated age in the past and decaying with timescales of $10^{7}, 3 \times 10^{7}, 10^{8}, 3 \times 10^{8}$ and $10^{9}$ years.

$\mathrm{c}$ The numbers in parentheses are the number of clusters (or complexes) measured in each region.

$\mathrm{d}$ The masses and ages for the interclump regions correspond to rectangles with areas of 255, 204, 270, and 609 pixels for $\mathrm{f}, \mathrm{g}, \mathrm{h}$, and $\mathrm{i}$, respectively. A constant star formation rate back to the indicated age was assumed.

e A constant star formation rate back to the indicated age was assumed.
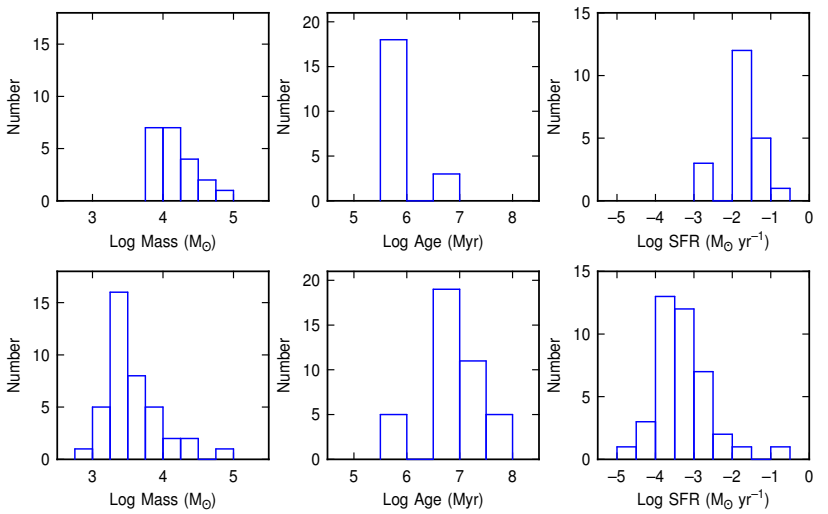

Figure 9. Mass, age, and star formation rate histograms for the star-forming clusters for the head (top) and tail (bottom) regions.

the size distribution of the star-forming regions in Kiso 5639 , we used the IRAF task gauss to Gaussian blur the F336W image by five successive factors of two. Then the program SExtractor was used to identify and count sources in each of these Gauss-blurred images. The result is a count of regions larger than the blurring size. A plot of the log-count versus the minimum log-size is given in Figure 11. The power law distribution from $4 \mathrm{pc}$ to $150 \mathrm{pc}$ follows from the hierarchical structure; here the slope is $-0.47 \pm 0.05$. For 12 spiral and irregular galaxies in Elmegreen et al. (2014), a similar procedure gave slopes of -0.76 to -1.86 using the LEGUS Legacy ExtraGalactic UV Survey (Calzetti et al. 2015). A shallow slope, like that found here, means that star formation is spread out somewhat uniformly. This is reasonable from

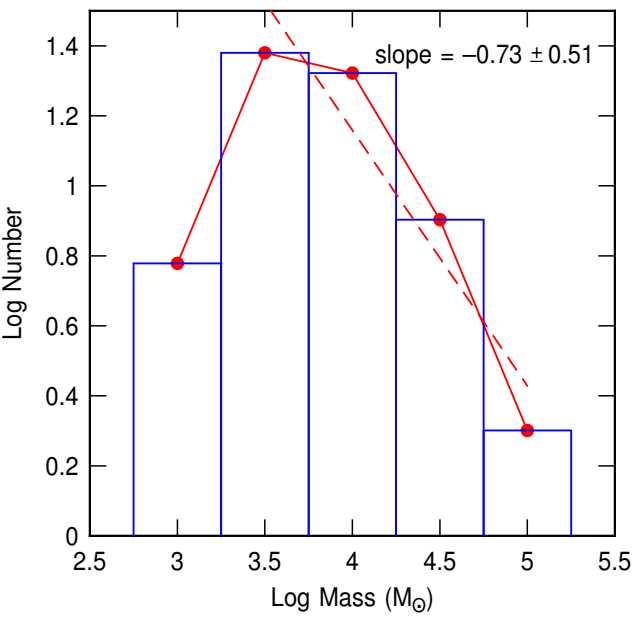

Figure 10. Mass histogram and power law fit for the 61 measured star-forming clusters in Kiso 5639.

Figure 4, where the main star-forming regions are spread broadly across the galaxy.

\subsection{Tadpole Intrinsic Shape}

The three-dimensional structure of Kiso 5639 is not easily determined from its projected shape, but the optical spectrum implies rotation (Sánchez Almeida et al. 2013). The F814W image also shows what may be a faint underlying disk, especially in the logarithmic stretch in Figure 4. Based on the assumption that the main disk is intrinsically round, we measured the outer isophotes of the major and minor axes of the F814W image to deter- 


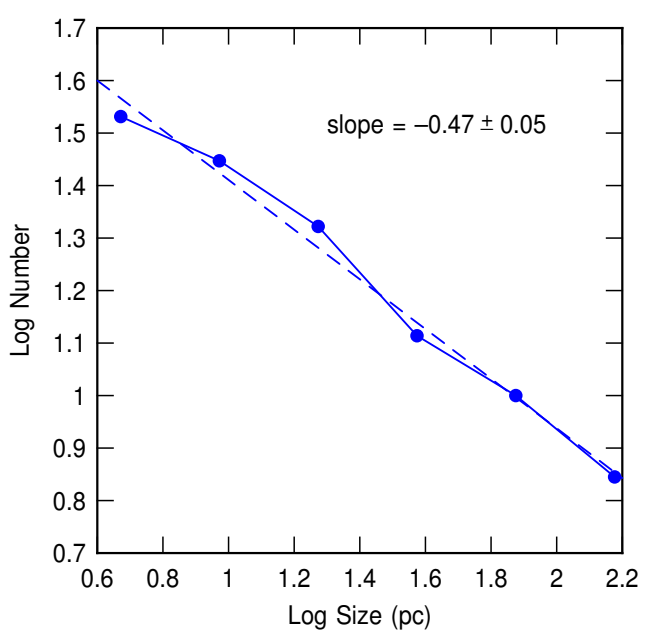

Figure 11. Histogram of number versus size of the star-forming clumps, as measured from SExtractor on images Gauss-blurred by $1,2,4,8,16,32$.

mine the orientation and inclination. The images were then rotated in IRAF by $-9.3^{\circ}$ using the task rotate and stretched by a factor of 2.67 (corresponding to an inclination of $68^{\circ}$ for a thin disk) using the task magnify. Figure 12 shows the deprojected color composite image for the galaxy using F438W (blue), F547M (green), and $\mathrm{F} 81 \mathrm{~W}$ (red) images. The head region is round in the inclined view and elongated in the deprojected view. The projected roundness suggests that the head is more threedimensional like a sphere than two-dimensional like a circle in the plane. Disk thickness elsewhere will cause another distortion, with one side of the minor axis showing slightly more stars from the back side of the disk and the other side showing slightly more from the front side. If we correct the apparent inclination, $i_{\text {appar }}$, for an intrinsic ratio of thickness to diameter equal to 0.2 using the equation $\cos i_{\text {true }}^{2}=\left(\cos i_{\text {appar }}^{2}-0.2^{2}\right) /\left(1-0.2^{2}\right)$ from Sánchez-Janssen et al. (2010), then the true inclination is $i_{\text {true }}=84^{\circ}$.

The assumption of a circular disk for the whole galaxy is reasonable considering axial ratios for oneclump galaxies in the Kiso sample of UV bright galaxies (Miyauchi-Isobe, Maehara, \& Nakajima 2010). If tadpoles are highly inclined disk galaxies, there should be face-on versions of tadpoles that have one dominant starforming clump in a round disk. Kiso galaxies labeled as I,c and I,g are galaxies identified as having one or several main clumps. There are 159 of these galaxies in the Sloan Digital Sky Survey (SDSS), which we examined to select one-clump galaxies. Some galaxies in these lists were clearly spiral or interacting, and were excluded. Of the remainder, 48 had one main clump, and 44 had several main clumps; some of the one-clump dominant galaxies were in the I,g category.

A histogram of the isophotal axial ratios for the 48 one-clump galaxies is shown in Figure 13 The distribution is fairly flat over most of the range, except for a spike at an axial ratio of 0.35 . This flatness means that most one-clump galaxies are approximately circular disks seen in random projections with tadpoles representing the edge-on cases (compare to similar distributions for clumpy galaxies in Elmegreen et al. 2005a b). The spike suggests that a small fraction of one-clump galaxies may

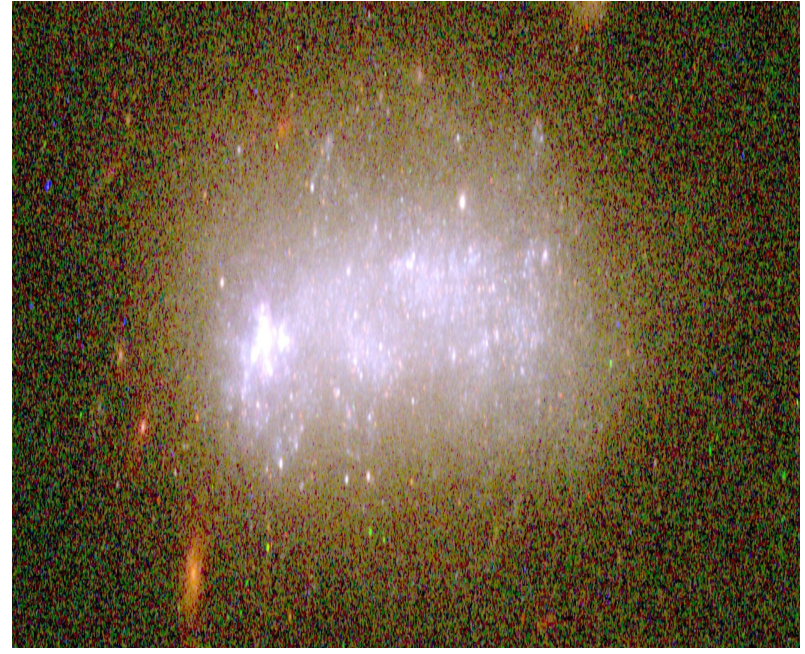

Figure 12. Deprojected image of F438W (blue), F547M (green) and $\mathrm{F} 814 \mathrm{~W}$ (red), assuming that the galaxy is round and using a rotation of $-9.3^{\circ}$ and an inclination of $68^{\circ}$ as determined from the outer F814W isophotes.

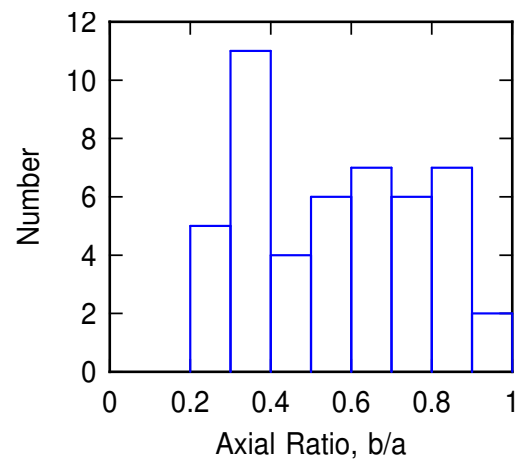

Figure 13. Axial ratios of galaxies from the Kiso ultraviolet survey with one main star-forming clump, measured from SDSS images.

be intrinsically elongated. For example, the galaxy I Zw 18 is an apparent tadpole composed of one large clump of star formation in a main body with a smaller one to the south in the same old-stellar envelope, and a third clump to the north, somewhat detached; it appears to be intrinsically elongated. The drop-off in the distribution of axial ratios below 0.2 in the figure is presumably the ratio of disk thickness to diameter. Small galaxies like this tend to be relatively thick (Elmegreen et al. 2005b; Sánchez-Janssen et al. 2010, Elmegreen \& Hunter 2015).

\subsection{Star-forming Head}

\subsubsection{Star Formation and Ionization Rates}

The $\mathrm{H} \alpha$ luminosity of the central $3^{\prime \prime}$ diameter region of the head ( $360 \mathrm{pc}$ ) is $8.0 \times 10^{39} \mathrm{erg} \mathrm{s}^{-1}$ according to the SDSS spectrum (Elmegreen et al. 2012). For the HST observations, the $\mathrm{H} \alpha$ luminosity of a core rectangular region centered on the head is $8.8 \pm 0.16 \times 10^{39} \mathrm{erg} \mathrm{s}^{-1}$. This $\mathrm{H} \alpha$ luminosity is determined from the continuumsubtracted $\mathrm{F} 657 \mathrm{~N} \mathrm{H} \alpha$ image, which comes from the $\mathrm{H} \alpha$ filter width, $121 \AA$, multiplied by the difference between the F657N intensity and the F547M intensity used for the continuum, and then multiplied by $4 \pi D^{2} c / \lambda^{2}$ (see footnote 6 above). For reference, the continuum-subtracted $\mathrm{H} \alpha$ apparent $\mathrm{AB}$ magnitude of the head is 15.99. The size 
of this region is effectively $3.6^{\prime \prime}$, calculated as the square root of the number of pixels in the rectangle multiplied by the pixel size. Assuming the conversion in equation (2) of Kennicutt (1998), the head luminosity from HST $\mathrm{H} \alpha$ corresponds to a SFR of $\sim 0.070 M_{\odot} \mathrm{yr}^{-1}$, which gives the $\log$ value of -1.2 in Table 2 .

The SFR determined from the ratio of the head region's photometric mass to its photometric age, $2.7 M_{\odot}$ $\mathrm{yr}^{-1}$, is much larger than the $\mathrm{H} \alpha$ rate. One reason for this may be that the photometric ages are too young compared to the overall duration of star formation in the region. This could be the result of a dominance by the youngest massive stars in the ultraviolet bands, or from patchy dust that preferentially obscures older stars. There could also be a component of $\mathrm{H} \alpha$ that is obscured or too extended to see in our data. Extinction has an important effect on the inferred photometric SFR. Higher extinction requires more stellar mass to give the same brightness, and a younger age to give the same color, and the combined effect pushes up the SFR. Varying the extinction in our models from 0 to 1 magnitude in the visible band increases the total photometric SFR for the 21 clusters in the head from $0.13 M_{\odot} \mathrm{yr}^{-1}$ to $1.2 M_{\odot} \mathrm{yr}^{-1}$. The average cluster value in Table $2,10^{-1.7}$ per cluster, corresponds to a total for all 21 clusters of $0.4 M_{\odot} \mathrm{yr}^{-1}$, in the middle of this range and consistent with the average extinction that we find for these clusters, which is 0.65 mag. We do not derive the SFR based solely on the FUV flux because that method needs to assume a star formation history, such as a constant rate. In what follows, we use the SFR from the $\mathrm{H} \alpha$.

For typical recombination in a photoionized region, the Lyman continuum photon ionization rate, $S$, in photons $\mathrm{s}^{-1}$, is proportional to the $\mathrm{H} \alpha$ luminosity in erg $\mathrm{s}^{-1}$ according to the conversion $S=7.3 \times 10^{11} L(H \alpha)$, again using equation (2) in Kennicutt (1998). This implies the ionization rate is $\sim 6.4 \times 10^{51}$ photons $\mathrm{s}^{-1}$. For an ionization rate from an 09 star of $3 \times 10^{48} \mathrm{~s}^{-1}$ (Vacca et al. 1996), the head of Kiso 5639 contains $\sim 2100$ O9 stars.

The summed mass of the 21 measured clusters in the head region is $4.0 \times 10^{5} M_{\odot}$. Considering a Chabrier IMF in the 0.2 solar metallicity models by Bruzual \& Charlot (2003), such a mass of young stars at 1 Myr age would be expected to have an ionization rate of $1.9 \times 10^{51} \mathrm{~s}^{-1}$; at 3 and $10 \mathrm{Myr}$, that would be 1.5 and 0.4 times $10^{51} \mathrm{~s}^{-1}$, respectively. The rate for the 1 Myr age is about $30 \%$ of what we observe. Alternatively, we can integrate an IMF that is flat below $0.5 M_{\odot}$ and a power law with the Salpeter slope of -2.35 above $0.5 M_{\odot}$ to $120 M_{\odot}$, and normalize it to the same total cluster mass. This gives $\sim 900$ stars more massive than $25 M_{\odot}$, the mass of an O9 star (Vacca et al. 1996); that is $\sim 40 \%$ of the number obtained from the total ionization rate. These fractions imply that $30 \%-40 \%$ of the O stars in the head of Kiso 5639 are in clusters; the rest are presumably between the clusters.

\subsubsection{Ionization and Confinement of the Head HII Region}

If the full diameter of the head, $7^{\prime \prime} \sim 830 \mathrm{pc}$, is the diameter of a Strömgren sphere formed by ionization at the inferred rate of $6.4 \times 10^{51}$ photons $\mathrm{s}^{-1}$, then the rms electron density is $n_{\mathrm{e}} \sim 1.7 \mathrm{~cm}^{-3}$, assuming a recombination coefficient $\alpha^{(2)}=2.6 \times 10^{-13} \mathrm{~cm}^{3} \mathrm{~s}^{-1}$. At a temper- ature $T=10^{4} \mathrm{~K}$, the corresponding pressure would be $P_{\mathrm{HII}}=2.1 n_{e} k_{\mathrm{B}} T=3.5 \times 10^{4} k_{\mathrm{B}}$ for Boltzmann's constant $k_{\mathrm{B}}$.

We are interested in whether this pressure is too large for the galaxy to retain the gas. If the pressure exceeds the self-gravitational pressure from the weight of the disk, then the HiI region will expand. For the observed $\mathrm{H} \alpha$ line half-width of $\sim 23 \mathrm{~km} \mathrm{~s}^{-1}$ (Sánchez Almeida et al. 2013), the expansion time over the radius of 415 pc is only $\sim 20$ Myr. A recent WIYN telescope spectrum taken by one of us (JSG) suggests that the $\mathrm{H} \alpha$ line $\sigma$ is $\sim 30 \mathrm{~km} \mathrm{~s}^{-1}$, which would make the expansion time $14 \mathrm{Myr}$. These high linewidths for the ionized gas also suggest that the HII region pressure is larger than $\sim 3.5 \times 10^{4} k_{\mathrm{B}}$ from the thermal motions alone. Gas with a density of $\rho=1.7 m_{\mathrm{H}} \mu \mathrm{cm}^{-3}$ for hydrogen mass $m_{\mathrm{H}}$, mean atomic weight $\mu=1.36$, and turbulent speed $\sigma \sim 30 \mathrm{~km} \mathrm{~s}^{-1}$ has a pressure $\rho \sigma^{2} \sim 2.5 \times 10^{5} k_{\mathrm{B}}$.

The self-gravitational pressure in a disk is approximately $P_{\text {dyn }}=(\pi / 2) G \Sigma_{\text {tot }}^{2}$ for total mass surface density $\Sigma_{\text {tot }}$, which consists of stars, gas, and some dark matter (Elmegreen 1989). The average surface density of old stars in the interclump regions is $\sim 25 \pm 5 M_{\odot} \mathrm{pc}^{-2}$, as obtained from SED fitting (Table 2). This is about the same as the surface density obtained from the galaxy dynamical mass, $1.5 \times 10^{8} M_{\odot}$ (Section 2 spread out over the galaxy diameter of $2.7 \mathrm{kpc}$, assuming the galaxy is a circular disk. That result is $26 M_{\odot} \mathrm{pc}^{-2}$, although it probably includes an amount of dark matter comparable to the stellar mass. The SED mass of the head without the background interclump emission subtracted (as it is in Table 1), is $3.1 \times 10^{6} M_{\odot}$ inside the area measured (8085 square pixels or $1.79 \times 10^{5} \mathrm{pc}^{2}$ ). This gives a total stellar surface density of $17.3 M_{\odot} \mathrm{pc}^{-2}$ in the head region. The dynamical mass in the $130 \mathrm{pc}$ diameter core of the head measured from the $\mathrm{H} \alpha$ velocity gradient (Sánchez Almeida et al. 2013) is $1.7 \times 10^{7} M_{\odot}$. This suggests a surface density of $1.3 \times 10^{3} M_{\odot} \mathrm{pc}^{-2}$, which is much larger than the photometric surface density. The difference could be the result of a component in the $\mathrm{H} \alpha$ velocity gradient that is caused by pressure forces, rather than gravity, or it could indicate a dense dark mass in this region, perhaps from a molecular cloud.

The photometric surface densities are within a factor of $\sim 2$ of the total mass surface density that would be needed to make the dynamical pressure equal to the HiI region thermal pressure, which is $\Sigma_{\text {tot }}=$ $\left([2 / \pi G] P_{\mathrm{HII}}\right)^{0.5} \sim 32 M_{\odot} \mathrm{pc}^{-2}$. For the HII region turbulent pressure of $2.5 \times 10^{5} k_{\mathrm{B}}$, the total mass surface density needed for binding is $\Sigma_{\text {tot }}=85 M_{\odot} \mathrm{pc}^{-2}$. The dynamical surface density of $10^{3} M_{\odot} \mathrm{pc}^{-2}$ is more than enough to confine the HII region.

The SFR determined from $\mathrm{H} \alpha$ is $\sim 0.070 M_{\odot} \mathrm{yr}^{-1}$ for the $3.6^{\prime \prime}$ square core $(430 \mathrm{pc})$ measured in the head. This gives a rate per unit area of $\Sigma_{\mathrm{SFR}}=0.38 M_{\odot}$ $\mathrm{pc}^{-2} \mathrm{Myr}^{-1}$. This rate exceeds an empirically determined limit for the formation of a superwind (Heckman 2002), which is $\sim 0.1 M_{\odot} \mathrm{pc}^{-2} \mathrm{Myr}^{-1}$, making it plausible that the HII region or hot gases associated with it are not bound to the galaxy.

\subsubsection{Enhanced Star Formation Rates}


An estimate for the molecular gas surface density may be made from the product of $\Sigma_{\mathrm{SFR}}$ and the typical molecular gas consumption time, which is $\tau_{\text {gas }} \sim 2$ Gyr in normal galaxy disks (Bigiel et al. 2008, Leroy et al. 2008) and the outer regions of spiral galaxies, as obtained by stacking $\mathrm{CO}$ spectra when the individual regions are too faint to see (Schruba et al. 2011). For $\Sigma_{\mathrm{SFR}}=0.38 M_{\odot}$ $\mathrm{pc}^{-2} \mathrm{Myr}^{-1}$ calculated above, the associated molecular surface density would be $760 M_{\odot} \mathrm{pc}^{-2}$. This is comparable to the dynamical surface density of $1.3 \times 10^{3} M_{\odot}$ $\mathrm{pc}^{-2}$ (Sect. 4.3.2). Atomic gas would increase this gas surface density even more. Possibly, the consumption time is shorter than the canonical value; $\tau_{\text {gas }}=66 \mathrm{Myr}$ would allow $\Sigma_{\text {gas }} \sim \Sigma_{\text {stars }} \sim 25 M_{\odot} \mathrm{pc}^{-2}$ for the observed SFR surface density. This short consumption time would place the head of Kiso 5639 a factor of $\sim 30$ above the normal Kennicutt-Schmidt relation between star formation surface density and gas surface density, and in the ULIRG regime (e.g., Sargent et al. 2014).

Enhanced SFRs per unit gas mass can also be inferred from our previous observations of 10 XMP galaxies (Sánchez Almeida et al. 2015). There we derived a ratio of $f_{\mathrm{Z}}=Z_{\text {burst }} / Z_{\text {host }}=0.3-0.1$ for metallicity in the starburst compared to the host, and a factor of $f_{\mathrm{SFR}}=\Sigma_{\mathrm{SFR}, \text { burst }} / \Sigma_{\mathrm{SFR} \text {,host }}=10-100$ for the star formation surface density enhancement. For the present galaxy, Kiso 5639, $f_{\mathrm{Z}}=0.4$ and $f_{\mathrm{SFR}}=40$ (Sánchez Almeida et al. 2013). Simulations by Ceverino et al. (2016) obtain similar factors. These factors place constraints on the relative efficiency of star formation in the burst and on the previous metallicity of the cosmic gas relative to the host, written as the factor $f_{\text {cosmic }}$. We assume that the starburst region contains gas column density contributions from the cosmic cloud, $\Sigma_{\text {cosmic }}$, and from the host, $\Sigma_{\text {host }}$, in some ratio $C$. Then

$$
f_{\mathrm{Z}}=\frac{f_{\text {cosmic }} \Sigma_{\text {cosmic }}+\Sigma_{\text {host }}}{\Sigma_{\text {cosmic }}+\Sigma_{\text {host }}}=\frac{f_{\text {cosmic }} C+1}{C+1} .
$$

We also assume that the SFR scales with gas column density to a power $\alpha$ but has an additional efficiency enhancement factor $E$ in the starburst compared to the host. Then

$$
f_{\mathrm{SFR}}=E(C+1)^{\alpha} .
$$

For the Kennicutt-Schmidt relation with total gas, $\alpha \sim$ 1.5 (Kennicutt \& Evans 2012). Setting $f_{\mathrm{Z}} \sim 0.4$ and $f_{\mathrm{SFR}} \sim 40$ as for Kiso 5639 and the galaxies in Sánchez Almeida et al. (2015), we find that if there is no enhancement in efficiency $(E=1)$ then the accreted gas surface density factor is $C=11$ and the ratio of accreted metallicity to host metallicity is $f_{\text {cosmic }}=0.34$. These numbers decrease as the enhancement increases: for $E=4$ to 10 , $C=3.6$ to 1.5 , and $f_{\text {cosmic }}=0.24$ to 0.005 . Unless the accreted gas surface density is significantly higher than the host gas surface density (i.e., unless $C$ is large), the enhancement above the normal KS relation $(E)$ has to be high and the relative metallicity of the accreted gas $\left(f_{\text {cosmic }}\right)$ has to be low.

The dense parts of individual molecular clouds (Lada et al. 2010) have relatively short consumption times like the small values implied by large $E$ (the relative consumption time is inversely proportional to $E$ ), but usually observations on large scales like the $\sim 830$ pc head region of Kiso 5639 have the canonical $\sim 2$ Gyr and no enhanced star formation (i.e., $E \sim 1$, Bigiel et al. 2008 Leroy et al. 2008). We conclude that the accretion of an amount of low metallicity gas comparable to or a few times larger than the previous gas column density in the disk triggered a substantial burst star formation with a relative short gas consumption time.

\subsubsection{Head Formation by Gravitational Instability}

The largest star formation complexes in many galaxy disks form by gravitational instabilities in the turbulent interstellar medium. We are interested in whether the expected size and mass of a region formed this way match the tadpole head in Kiso 5639. The characteristic mass for an instability depends on the gas velocity dispersion and the disk column density. For a cosmological context with active accretion, Ceverino et al. (2010) suggest that the clump mass, $M_{\mathrm{c}}$, formed by such an instability is related to the disk mass, $M_{\mathrm{d}}$, as $M_{\mathrm{c}}=0.27 \delta^{2} M_{\mathrm{d}}$ where $\delta=M_{\mathrm{d}} / M_{\text {total }}$ and $M_{\text {total }}$ is the total disk mass out to the radius of the clump. They also suggest the clump radius is $R_{\mathrm{c}} \sim 0.52 \delta R_{\mathrm{d}}$.

For Kiso 5639, the head region could be a clump that formed by a gravitational instability after fresh gas accreted in this part of the galaxy. To compare the observations with this theoretical prediction, the clump mass is taken to be twice the total SED mass of the head measured without interclump background subtraction, or $M_{\mathrm{c}} \sim 6.2 \times 10^{6} M_{\odot}$. The factor of 2 accounts for gas that we do not detect. The disk mass is assumed to be half of the dynamical mass because of dark matter contributing to the rotation curve; then $M_{\mathrm{d}} \sim 0.75 \times 10^{8} M_{\odot}$ and $\delta=0.5$. Thus, the theory in Ceverino et al. (2010) predicts that $M_{\mathrm{c}}=0.07 M_{\mathrm{d}}$, and the observations agree, with $M_{\mathrm{c}} \sim 0.08 M_{\mathrm{d}}$. Also for $\delta=0.5$, the theory predicts that the clump size is $25 \%$ of the galaxy size, and that is also about right for the head.

\subsubsection{Holes and Feedback in the Head HII region}

Holes are identified in the continuum-subtracted $\mathrm{H} \alpha$ image as low emission regions surrounded by ring-like structures. The holes evident in Figure 14 (top) are marked by red ellipses in the bottom panel. They span diameters of 28 to $100 \mathrm{pc}$, as listed in Table 3 . Hydrodynamic calculations of supergalactic winds show that holes and filamentary structures can develop in the presence of a high concentration of super star clusters, such as those observed in M82 (Tenorio-Tagle, Silich, \& Muñoz-Tuñón 2003). The holes and filaments in Kiso 5639 could have the same origin.

The cavities in the head of Kiso 5639 could form by a combination of stellar winds and supernovae, depending on the age. Stellar winds would have to dominate if this is a very young region, as suggested by the photometry. With an ambient density of 1 atom $\mathrm{cm}^{-3}$ as calculated above for the HII region, the wind luminosity $L_{33}$, in units of $10^{33}$ ergs $\mathrm{s}^{-1}$, and the wind duration $t_{\mathrm{My}}$, in units of one million years, that are required to make a wind-swept bubble $50 \mathrm{pc}$ in radius satisfy the product $L_{33} t_{\mathrm{Myr}}^{3}=2.5 \times 10^{4}$, according to equation (6) in Castor et al. (1975). An O9 star with solar metallicity has a mass loss rate of $\dot{M} \sim 10^{-7.5}$ and wind speed of $\sim 2000$ $\mathrm{km} \mathrm{s}^{-1}$ (Martins 2015), so $L_{33}=40$. The mass loss rate decreases with metallicity as $Z^{0.83}$ (Martins 2015), 
Table 3

$\mathrm{H} \alpha$ Holes in Head

\begin{tabular}{|c|c|c|c|c|c|c|}
\hline Number & $\begin{array}{c}\mathrm{RA} \\
(\mathrm{J} 2000)\end{array}$ & $\begin{array}{c}\text { Dec } \\
\text { J2000 }\end{array}$ & x diameter & y diameter & $\begin{array}{l}\mathrm{x} \text { diameter } \\
\mathrm{pc}\end{array}$ & $\begin{array}{c}\text { y diameter } \\
\text { pc }\end{array}$ \\
\hline 1 & 11:41:07.700 & $+32: 25: 37.70$ & 0.57 & 0.85 & 67.5 & 100.9 \\
\hline 2 & 11:41:07.689 & $+32: 25: 36.20$ & 0.57 & 0.47 & 67.6 & 55.6 \\
\hline 3 & $11: 41: 07.596$ & $+32: 25: 37.95$ & 0.34 & 0.23 & 40.0 & 27.0 \\
\hline 4 & $11: 41: 07.598$ & $+32: 25: 36.94$ & 0.61 & 0.50 & 72.7 & 59.4 \\
\hline 5 & $11: 41: 07.583$ & $+32: 25: 35.74$ & 0.53 & 0.34 & 63.5 & 40.9 \\
\hline 6 & $11: 41: 07.606$ & $+32: 25: 35.17$ & 0.31 & 0.27 & 36.9 & 32.1 \\
\hline 7 & $11: 41: 07.451$ & $+32: 25: 38.16$ & 0.61 & 0.43 & 72.9 & 51.2 \\
\hline 8 & $11: 41: 07.420$ & $+32: 25: 37.61$ & 0.23 & 0.24 & 27.8 & 28.3 \\
\hline 9 & $11: 41: 07.469$ & $+32: 25: 36.97$ & 0.45 & 0.37 & 53.4 & 44.2 \\
\hline 10 & $11: 41: 07.469$ & $+32: 25: 36.26$ & 0.55 & 0.34 & 65.3 & 40.2 \\
\hline
\end{tabular}

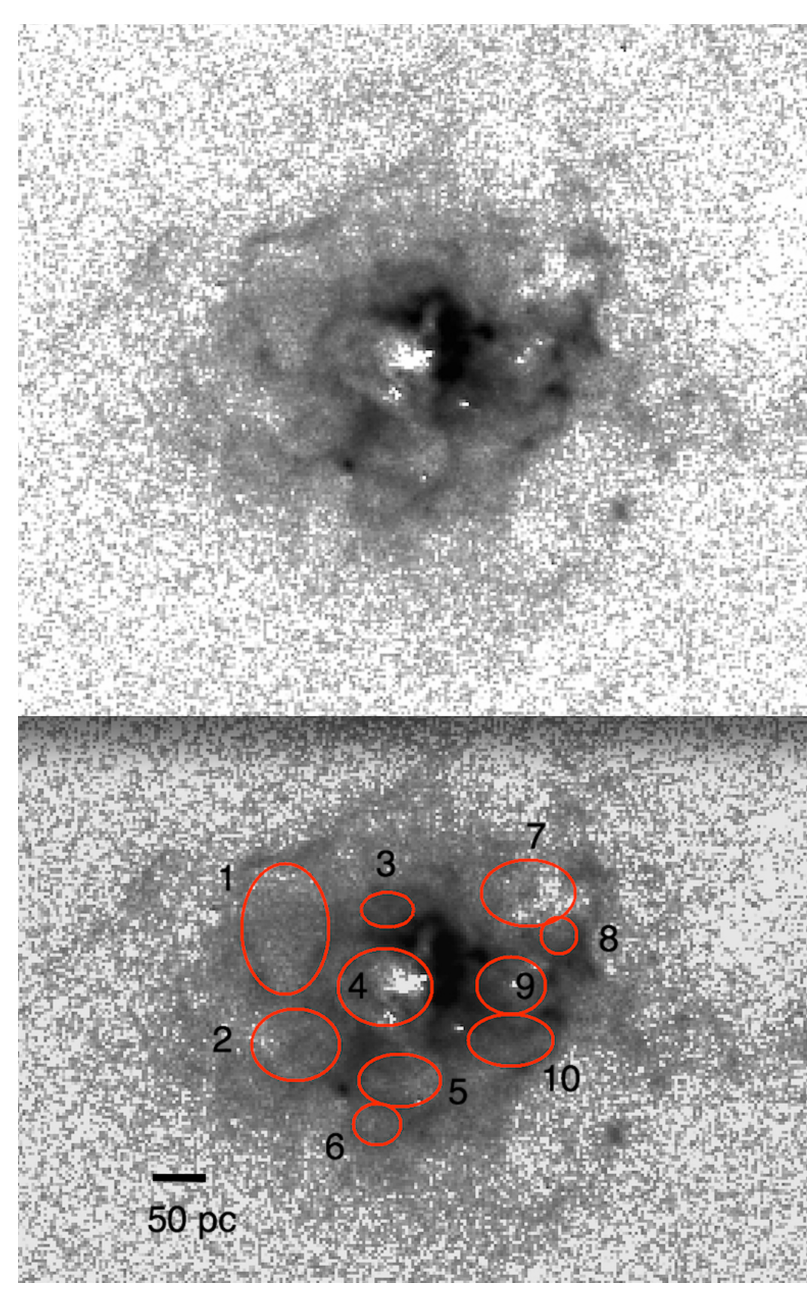

Figure 14. (top) Enlarged logarithmic $\mathrm{H} \alpha$ continuum-subtracted image showing the head. (bottom) Same as top, with ellipses outlining the main holes in the head. Numbers correspond to the listing in Table 3

where $Z \sim 0.1$ for the head of Kiso 5639, making $L_{33}=$ 6. Thus, an equivalent of $\sim 4000$ O9 stars would be needed for each large bubble. An O5 star has about 100 times the mass loss rate of an O9 star (Martins 2015), so 40 equivalent O5 stars would be needed to evacuate the cavities with stellar winds alone. These numbers of $\mathrm{O}$ stars produce between $1.5 \times 10^{52}$ and $1.4 \times 10^{51}$ ionizing photons $\mathrm{s}^{-1}$, respectively (Vacca et al. 1996), which is consistent with the observed rate of $6.4 \times 10^{51} \mathrm{ph} \mathrm{s}^{-1}$.
Multiple supernovae should help clear the cavities if the age is older than a few Myr. If we consider that a single supernova deposits a mechanical energy of 1 to 10 $\times 10^{49}$ ergs to the surrounding gas (assuming 1\% to $10 \%$ efficiency, Walch \& Naab 2015), and that the supernova rate is $S$ supernovae per million years, then the average supernova mechanical luminosity is $3-30 \times 10^{35} \mathrm{~S}$ erg $\mathrm{s}^{-1}$ or $(300-3000) S L_{33}$. Setting this to $2.5 \times 10^{4}$ for a 1 million year duration in the Castor et al. solution above, we get a number of supernova $S$ equal to 8-80 to make a $100 \mathrm{pc}$ diameter bubble in that time. For a 3 Myr duration, the number is smaller by $3^{3}=27$. These are reasonable numbers if the duration of star formation is long enough to have had supernovae.

The hole morphology of the head region and the peripheral wispy $\mathrm{H} \alpha$ emission are similar in appearance to the holes and wisps in I Zw 18 (see Figs. 1 and 2 in Hunter \& Thronson 1995). I Zw 18 is one of the lowest metallicity local galaxies (Searle \& Sargent 1972, Izotov et a. 2009). Our estimates for supernovae clearing in Kiso 5639 are consistent with similar conclusions about star formation in I Zw 18 based on HST WFPC2 images (Hunter \& Thronson 1995, Annibali et al. 2013. Lebouteiller et al. 2013).

Supernova clearing of cavities may also lead to gas expulsion from the whole galaxy. Many of the shells in Figure 14 appear to be broken around the periphery of the head, suggesting a release of hot gas into the halo. Considering the low escape speed from this galaxy, $\sim 50$ $\mathrm{km} \mathrm{s}^{-1}$ (Sánchez Almeida et al. 2013), and the high thermal speed of a $10^{6} \mathrm{~K}$ supernova cavity, $\sim 80 \mathrm{~km} \mathrm{~s}^{-1}$, localized break out from the head should mean that a sizeable fraction of the cavity gas escapes from the disk region. This gas should ultimately become enriched with heavy elements, and if the escaping gas is preferentially enriched, then the metallicity in the remaining gas and stars can remain small. This is in accord with current models for the origin of the mass-metallicity relation in galaxies (e.g., Peeples \& Shankar 2011; Davé et al. 2012, Lilly et al. 2013; Fu et al. 2013).

\subsubsection{Clustering Fraction of Star Formation}

There are two indications that a high fraction of star formation in the head of Kiso 5639 is clustered, rather than dispersed. The first was mentioned above, namely that the summed mass of the measured clusters, $4.0 \times 10^{5} M_{\odot}$, produces $30 \%-40 \%$ of the $\mathrm{H} \alpha$ flux that is observed.

The second indication comes from the summed cluster 
masses in the head relative to the total young stellar mass in the head, which is $10^{6.2 \pm 0.12} M_{\odot}$ (Table 2. The ratio is $25 \%$, but this ignores smaller clusters. We can integrate over the cluster mass function below the detection limit to include those smaller clusters. The cluster mass function is $N(M)=N_{0} M^{-1.73 \pm 0.51}$ from Section 4.1. We normalize this to the total count by setting the number greater than $10^{4} M_{\odot}$ equal to the observed number of 14 . This gives $N_{0}=0.73 \times 14 \times 10^{4 \times 0.73}=8.5 \times 10^{3} M_{\odot}^{0.73}$. Then the total integral of cluster mass from a lower limit of $10 M_{\odot}$ to $10^{4} M_{\odot}$ is $\int_{10}^{10^{4}} M N_{0} M^{-1.73} d M=$ $3.2 \times 10^{5} M_{\odot}$. This can be added to the observed mass above $10^{4} M_{\odot}$, which is $4.0 \times 10^{5} M_{\odot}$, to give a total estimated cluster mass above $10 M_{\odot}$ of $7.2 \times 10^{5} M_{\odot}$. This total is $45 \%$ of the young stellar mass.

The high fraction of clustering in the head of Kiso 5639 is inconsistent with the general lack of clusters in dwarf Irregular galaxies of the same total mass (Billett et al. 2002), but comparable to the clustering fraction in other starburst dwarfs like NGC 1569 and IC 1705, and in massive active and interacting galaxies (Adamo et al. 2015 Adamo \& Bastian 2015). High clustering suggests high pressures (Kruijssen et al. 2012), and highlights the peculiar nature of the head region studied here.

There are 8 clusters within $50 \mathrm{pc}$ of the center of the head, so the mean separation in $3 \mathrm{D}$ between them is about $100 / 8^{1 / 3}=50$ pc. The IRAF task imexamine was used to measure the radial profiles of the central clusters to determine their radii at half maximum intensity. This radius averaged $2 \mathrm{px}$, which is essentially the resolution limit, so the maximum diameter of the central clusters is $\sim 20 \mathrm{pc}$. Thus, the volume filling fraction of the clusters is $(20 / 50)^{3}=6 \%$. These clusters have a total mass of $2.5 \times 10^{5} M_{\odot}$, giving a mass density in the central $50 \mathrm{pc}$ of $0.5 M_{\odot} \mathrm{pc}^{-3}$, which is equivalent to 14 atoms $\mathrm{cm}^{-3}$.

We can compare the density of young clusters in Kiso 5639 with other starburst galaxies. In the densest clustered region of M82, there are 86 clusters in a $242 \times 242$ $\mathrm{pc}^{2}$ area, which is 1470 clusters per $\mathrm{kpc}^{2}$; in NGC 253, the peak projected cluster density is 113 per kpc ${ }^{2}$ (Melo et al. 2005). In Kiso 5639, the 8 clusters in $50 \times 50 \mathrm{pc}^{2}$ corresponds to 3200 per $\mathrm{kpc}^{2}$, which is twice that in M82. So the central region of Kiso 5639 has a high cluster density. However, the cluster masses in Kiso 5639 are not as large as in M82 where 3 clusters of $\sim 6 \times 10^{5} M_{\odot}$ each are within 25 pc or each other (Westmoquette et al. 2014), giving a combined mass density of $\sim 100 M_{\odot} \mathrm{pc}^{-3}$.

\subsection{Associated HI gas}

The only Hi data available for Kiso 5639 are at low resolution. In an Arecibo Hi survey of star-forming dwarfs, there is a detection near Kiso 5639 with $\log M\left(H_{\mathrm{I}}\right)=8.5$, where the mass is given in $M_{\odot}$ (Salzer et al. 2002). The HIJASS survey from the Lovell telescope, with a beamsize of 12 arcmin, had a detection in the region of Kiso 5639 in a common Hi halo that included dwarf galaxies Mrk 0746, Was 28, and SDSSJ114135.98+321654.0, with $\log M\left(H \mathrm{I}, M_{\odot}\right)=9.2$ (Wolfinger et al. 2013). The total Hi mass can be attributed to one, some, or all of these galaxies. The Hi halo shows a small extension to the Northwest, in the direction of Kiso 5639. There is no optical evidence that these galaxies are interacting.

In the 45 arcsec resolution NRAO VLA Sky Survey
(NVSS) by Condon et al. (1998), there is a $21 \mathrm{~cm}$ radio continuum detection with a flux of $2.7 \mathrm{mJy}$ slightly southwest of the optical position of Kiso 5639 (the starforming head). The diffuse nature of the weak emission indicates that it is likely related to star formation. It is possible that the Hi emission is a reservoir from which accreted gas is stimulating the current star formation.

If the Lyman continuum escape fraction from the starforming head were comparable to that estimated for Ly $\alpha$ emitting galaxies, which is $25 \%$ or more (Blanc et al. 2011; Zheng et al. 2012), then the ionization rate in the surrounding gas would be $\sim 1.6 \times 10^{51} \mathrm{~s}^{-1}$, based on the observed rate inside the head of $6.4 \times 10^{51} \mathrm{~s}^{-1}$ given above. The Strömgren radius for this ionization could extend to $1 \mathrm{kpc}$ if the average peripheral density is 0.22 $\mathrm{cm}^{-1}$. This $\mathrm{kpc}$ extent would subtend an angle of $8.4^{\prime \prime}$ in all directions and be easily observed; it is $3 / 4$ of the radius of the galaxy. The only evidence for peripheral ionization that we see in Figures 2 and 3 is the system of filaments extending away from the body of the galaxy. Their lengths are typically $1.5-2^{\prime \prime}$ or $\sim 150-250 \mathrm{pc}$.

On the other hand, recent estimates of the Lyman continuum escape fraction for low mass star-forming galaxies at $\mathrm{z} \sim 1$ based on HST grism spectroscopy plus GALEX FUV indicate escape fractions of less than $2 \%$ (Rutkowski et al.2016), which would not be sufficient to illuminate external gas.

\section{CONCLUSIONS}

HST WFC3 observations of the local metal-poor galaxy Kiso 5639 show a large star-forming region 830 pc in diameter with 21 clusters of average mass $\sim 2 \times$ $10^{4} M_{\odot}$ and an $\mathrm{H} \alpha \mathrm{SFR}$ of $0.070 M_{\odot} \mathrm{yr}^{-1}$. The overall galaxy appears to be a rotating disk with an inclination of $68^{\circ}-84^{\circ}$ (depending on thickness), but the star-forming region at one end makes it look like a tadpole or comet. Older and less massive clusters are in four other regions in the tail.

The metallicity in the large starburst region was previously found to be about $40 \%$ of that in the rest of the galaxy, suggesting that the burst occurred in recently accreted low-metallicity gas. A reservoir of HI associated with the galaxy has a mass comparable to the dynamical mass in the optical disk. This HI could be the source of the accreting gas, or both the HI and the galaxy disk could be accreting together from a common extragalactic source.

The properties of the burst region indicate an unusual, high pressure event. The gas depletion time is shorter than in spiral galaxies by a factor of $5-30$, depending on the accreting mass, and the clustering fraction of star formation is high, $30-45 \%$. $\mathrm{H} \alpha$ observations show 10 cavities in the tadpole head with sizes of 20-100 pc, which could each arise from many dozens of supernovae over a period of several Myr. The perimeter of the galaxy shows broken $\mathrm{H} \alpha$ shells and filamentary structures that include some star formation.

We conclude that the accretion of an amount of low metallicity gas that is comparable to or a few times larger than the previous gas column density in the disk of the low-metallicity dwarf galaxy Kiso 5639 triggered a substantial burst of star formation in one localized region, and that star formation there has a relative short gas consumption time and a high clustering fraction. 
Acknowledgments: We thank NASA and STScI for observing time and grant support. DME is supported by HST-GO-13723.002-A and BGE is supported by HSTGO-13723.001-A; both are grateful to the Severo Ochoa and Jesús Serra Foundation for support during a visit to the Instituto de Astrofísica de Canarias. The work of JSA, CMT, and MF has been partly funded by the Spanish Ministry of Economy and Competitiveness, project AYA2013-47742-C4-2-P. JMA acknowledges support from the European Research Council Starting Grant (SEDmorph; P.I. V. Wild). MR acknowledges support from an appointment to the NASA Postdoctoral Program at Goddard Space Flight Center. DC acknowledges support from the European Research Council Advanced Grant (STARLIGHT; P.I. Ralf Klessen) This research has made use of the NASA/IPAC Extragalactic Database (NED) which is operated by the Jet Propulsion Laboratory, California Institute of Technology, under contract with the National Aeronautics and Space Administration.

\section{REFERENCES}

Adamo, A., Kruijssen, J. M. D., Bastian, N., Silva-Villa, E., \& Ryon, J. 2015, MNRAS, 452, 246

Adamo A., \& Bastian N., 2015, in The Birth of Star Clusters, ed. Stahler S.W., Springer, arXiv151108212

Annibali, F., Cignoni, M., Tosi, M., van der Marel, R., Aloisi, A., Clementini, G., Contreras Ramos, R., Fioentino, G., Marconi, M., \& Musella, I. 2013, AJ, 146, 144

Bigiel, F., Leroy, A., Walter, F., Brinks, E., de Blok, W. J. G. Madore, B., \& Thornley, M. D. 2008, AJ, 136, 2846

Billett, O.H., Hunter, D.A., Elmegreen, B.G. 2002, AJ, 123, 1454

Blanc, G. Adams, J., Gebhardt, K., et al. 2011, ApJ, 736, 31

Bruzual, G., \& Charlot, S. 2003, MNRAS, 344, 1000

Cairós, L., et al. 2001b, ApJS, 136, 393

Calzetti, D., Armus, L., Bohlin, R.C., Kinney, A.L., Koornneef, J., \& Storchi-Bergmann, T. 2000, ApJ, 533, 682

Calzetti, D., Lee, J., Sabbi, E., Adamao, A., et al. 2015, AJ, 149, 51

Castor, J., McCray, R. \& Weaver, R. 1975, ApJ, 200, L107

Ceverino, D., Dekel, A. Bournaud, F. 2010, MNRAS, 404, 2151

Ceverino, D., Jorge Sánchez Almeida, J., Muñoz-Tuñón, C.,

Dekel, A., Elmegreen, B.G., Elmegreen, D.M., \& Primack, J. 2016, MNRAS, 457, 2605

Condon, J., Cotton, W., Greisen, E., Yin, Q., Perley, R., \& Broderick, J. 1998, AJ, 115, 1693

Davé, R., Finlator, K., \& Oppenheimer, B. D. 2012, MNRAS, 421, 98

Elmegreen, B.G. 1989, ApJ, 338, 178

Elmegreen, B.G., Elmegreen, D.M., 2005, ApJ, 627, 632

Elmegreen, B.G. 2010, in IAUS266, Star clusters: basic galactic building blocks throughout time and space, ed. R. de Grijs and J. Lepine, Cambridge University Press, p.3

Elmegreen, B. G., \& Elmegreen, D. M. 2010, ApJ, 722, 1895

Elmegreen, B.G., \& Hunter, D.A. 2015, ApJ, 805, 145

Elmegreen, D.M., Elmegreen, B.G., Rubin, D.S., \& Schaffer, M.A. 2005, ApJ, 631, 85

Elmegreen, D.M. Elmegreen, B.G., Adamo, A., Aloisi, A., Andrews, J., Annibali, F., Bright, S., Calzetti, D., Cignoni, M., Evans, A., Gallagher, J., Gouliermis, D., Grebel, E., Hunter, D., Johnson, K., Kim, H., Lee, J., Sabbi, E., Smith, L., Thilker, D., Tosi, M., \& Ubeda, L. 2014, ApJL, 787, L15

Elmegreen, D.M., Elmegreen, B.G., Sańchez Almeida, J., Muñoz-Tuñón, C., Putko, J., \& Dewberry, J. 2012, ApJ, 750, 95 Elmegreen, D. M., Elmegreen, B. G., Ravindranath, S., \& Coe, D. A. 2007, ApJ, 658, 763

Elmegreen, D. M., Elmegreen, B. G., Rubin, D. S., \& Schaffer, M. A. 2005, ApJ, 631, 85

Filho, M. E., Sańchez Almeida, J., Muñoz-Tuñón, C. et al. 2015, ApJ, 80282

Filho, M. E., Sańchez Almeida, J., Muñoz-Tuñón, C., Elmegreen, B., \& Elmegreen, D. 2015, ApJ, submitted

Filho, M., Winkel, B., Sańchez Almeida, J., Aguerri, J., Amorin, R., Ascasibar, Y., Elmegreen, B., Elmegreen D., Gomes, J., Humprey, A., Lagos, P., Morales-Luis, A., Muñoz-Tuñón, C., Papaderos, P., \& Vilchez, J. 2013, A\&A, 558, 18

Fu, J., Kauffmann, G., Huang, M.-l., et al. 2013, MNRAS, 434, 1531
Gil de Paz, A., Madore, B. F., \& Pevunova, O. 2003, ApJS, 147,

Heckman, T. 2002, in Extragalactic Gas at Low Redshift, ASP

Conference Proceedings Vol. 254. Edited by John S. Mulchaey and John Stocke. San Francisco: ASP, p.292

Huang, M-L., Kauffmann, G., Chen, Y.-M., Moran, S.M., Heckman, T.M., Davé, R., Johansson, J. 2013, MNRAS, 431, 2622

Hunter, D., \& Thronson, H. 1995, ApJ, 452, 238

Izotov, Y.I., Guseva, N., Fricke, K., \& Papderos, P. 2009, A\&A, 503,61

Kennicutt, R., C. 1998, ARAA, 36, 189

Kennicutt, R.C., \& Evans, N.J. 1 2012, ARA\&A, 50, 531

Kniazev, A. Y., Pustilni, S. A., Ugryumov, A. V., \& Pramsky, A. G. 2001, A\&A, 371, 404

Kruijssen, J. M. D. 2012, MNRAS, 426, 3008

Lada, C.J., Lombardi, M., \& Alves, J.F. 2010, ApJ, 724, 687

Larsen, S. S. 2009, A\&A, 494, 539

Lebouteiller, V., Heap, S., Hubeny, I., \& Kunth, D. 2013, A\&A, $553, \mathrm{~A} 16$

Leitherer, C., Li, I.-H., Calzetti, D., Heckman, T.M. 2002, ApJS, 140,303

Leroy, A.K., Walter, F., Brinks, E., Bigiel, F., de Blok, W. J. G., Madore, B., \& Thornley, M. D. 2008, AJ, 136, 2782

Lilly, S. J., Carollo, C. M., Pipino, A., Renzini, A., \& Peng, Y. 2013, ApJ, 772, 119

Loose, H.-H., \& Thuan, T. X. 1985, in Star-Forming Dwarf Galaxies and Related Objects, ed. D. Kunth, T. X. Thuan, and J. T. T. Vân (Gif-sur-Yvette: Edition Frontières), 73

MacKenty, J. W. \& Smith, L. 2012, CTE White Paper, Tech. rep., STScI

Markarian, B. E. 1969, Astrofizika, 5, 206

Martins, F. 2015, in SF2A-2015: Proceedings of the Annual meeting of the French Society of Astronomy and Astrophysics. Eds.: F. Martins, S. Boissier, V. Buat, L. Cambrésy, P. Petit, Société Francaise d'Astronomie et d'Astrophysique, pp.343-348

Mastropietro, C., Burkert, A., \& Moore, B. 2009, MNRAS, 399, 2004

Melo, V., Muñoz-Tuñón, C., Maíz-Appelániz, J., \& Tenorio-Tagle, G. 2005, ApJ, 619, 270

Miyauchi-Isobe, N., Maehara, H., \& Nakajima, K. 2010, Pub.Nat.Astro.Ob.Japan, 13, 9

Morales-Luis, A. B., Sánchez Almeida, J., Aguerri, J. A. L. \& Muñoz-Tuñón, C. 2011, astro-ph arXiv:1109.0235 v1

Noeske, K., Guseva, N., Frick, K., Izotov, Y., Papaderos, P., \& Thuan, T. 2000, A\&A, 361, 33

Rafelski, M., Teplitz, H. I., Gardner, J. P., et al. 2015, AJ, 150, 31

Papaderos, P., Guseva, N. G., Izotov, Y. I., \& Fricke, K. J. 2008, A\&A, 491, 113

Peeples, M. S. \& Shankar, F. 2011, MNRAS, 417, 2962

Pérez-Montero, E. 2014, MNRAS, 441, 2663

Rafelski, M., Teplitz, H., Gardner, J., Coe, D., et al. 2015, AJ, 150,31

Rutkowski, M., Scarlata, C., Haardt, F., Siana, B., Rafelski, M., et al. 2016, ApJ, 819, 81

Salzer, J., Rosenberg, J., Weisstein, E., Mazzarella, J., \& Bothun, G. 2002, AJ, 124, 191

Sánchez Almeida, J., Muñoz-Tuñón, C. Elmegreen, D.

Elmegreen, B., \& Mendez-Abreu, J. 2013, ApJ, 767, 74

Sánchez Almeida, J. Morales-Luis, A.B., Muñoz-Tuñón, C. Elmegreen, D.M., Elmegreen B.G., \& Mendez-Abreu, J. 2014a, ApJ, 783, 45

Sánchez Almeida, J., Elmegreen, B.G., Muñoz-Tuñón, C. \& Elmegreen, D.M. 2014b, A\&AR, 22, 71

Sánchez Almeida, J. Elmegreen, B.G., Munoz-Tunon, C., Elmegreen D.M., Perez-Montero, E., Amorin, R., Filho, M.E., Ascasibar, Y., Papaderos, P., \& Vilchez, J.M. 2015, ApJL, 810L, 15

Sánchez-Janssen, R., Méndez-Abreu, J., Aguerri, J. A. L. et al. 2010, MNRAS, 406, 65

Sargent, M. T., Daddi, E., Béthermin, M., et al. 2014, ApJ, 793, 19

Schruba, A., Leroy, A. K., Walter, F., et al. 2011, A.J, 142, 37

Searle, L., \& Sargent, W. 1972, ApJ, 173, 25

Straughn, A. N., Cohen, S. H., Ryan, R. E., Hathi, N. P., Windhorst, R. A., \& Jansen, R. A. 2006, ApJ, 639, 724

Tenorio-Tagle, G., Silich, S., \& Muñoz-Tuñón, C. 2003, ApJ, 597, 279

Vacca, W.D., Garmany, C.D., \& Shull, J.M. 1996, ApJ, 460, 914

van den Bergh, S., Abraham, R. G., Ellis, R. S., Tanvir, N. R.,

Santiago, B. X., \& Glazebrook, K. G. 1996, AJ, 112, 359

Verbeke, R., De Rijcke, S., Koleva, M., et al. 2014, MNRAS, 442, 1830

Walch, S., \& Naab, T. 2015, MNRAS, 451, 2757 
Westmoquette, M. S., Bastian, N., Smith, L. J., Seth, A. C., Gallagher, J. S., III, O'Connell, R. W., Ryon, J. E., Silich, S., Mayya, Y. D., Muñoz-Tuñón, C., \& Rosa González, D. 2014, ApJ, 789, 94

Windhorst, R. A. et al. 2006, New Astron. Rev., 50, 821

Wolfinger, K., Kilborn, V., Koribalski, B., Minchin, R., Boyce, P.,

Disney, M., Lang, R., \& Jordan, C. 2013, MNRAS, 428, 1790
Zheng, Z.-Y., Malhotra, S., Wang, J.-X., Rhoads, J. et al. 2012, ApJ, 746, 28 